\title{
Electromagnetic field measurements in ULF-ELF-VLF [0.001 Hz-100 KHz] bands
}

\author{
P. Palangio, F. Masci, M. Di Persio, and C. Di Lorenzo \\ Istituto Nazionale di Geofisica e Vulcanologia, Italy
}

Received: 10 June 2007 - Revised: 25 September 2007 - Accepted: 26 September 2007 - Published: 2 January 2008

\begin{abstract}
We are reporting the technological and scientific objectives of the MEM project. The MEM project has been activated in the INGV Observatory of L'Aquila to create in Central Italy a network of observatories in order to monitoring the electromagnetic signals in the frequency band $[0.001 \mathrm{~Hz}-100 \mathrm{kHz}]$. Some examples of the instrumentation developed in the frame of the project are reported. An innovative technique, based on the wide band interferometry is proposed to obtain detailed information concerning the several detected electromagnetic sources. Moreover, data from each station will be elaborated to investigate different sectors as the structure of ground electric conductibility, the electromagnetic phenomena connected with seismic activity, the separation of the electromagnetic fields originated in the Earth's interior and the electromagnetic phenomena originated in the magnetosphere, in the ionosphere and in the Earth-ionosphere cavity.
\end{abstract}

\section{Introduction}

The MEM Project (Magnetic and Electric fields Monitoring) has been activated in the INGV (Italian Istituto Nazionale di Geofisica e Vulcanologia) Observatory of L'Aquila since 2004 (Palangio et al., 2007). The leader partner of the project is the Italian Abruzzo Region. The other partners are the INGV Observatory of L'Aquila, the Regional Environmental Agency of Molise Region (ARPA-Molise), Italy, the University of Ferrara, Italy, the University of Tirana, Albania and the Geomagnetic Institute of Grocka, Beograd, Serbia. The main target of the MEM project is the creation in Central Italy of a permanent observatories network for the monitoring of the electromagnetic signals. Data from the network stations will allow us to realize environmental electromag-

Correspondence to: F. Masci

(masci@ingv.it) netic tomography through the representation of the electromagnetic fields distribution in the time, in the frequency and in the space domains. One of the scientific purposes of the MEM project is the electromagnetic monitoring of the geodynamical processes related to the tectonic activity (Teisseyre and Ernst, 2002). The other objectives are: the characterization of the electromagnetic background noise in the $[0.001 \mathrm{~Hz}-100 \mathrm{kHz}]$ frequency band in the Earth-ionosphere cavity; the realization of a model of the electrical conductivity of the Earth's crust; the study of the electromagnetic signals produced in the Earth's interior (Fraser-Smith et al., 1990). The technological objectives of the project are the development of new instruments and the know-how transfer to the industry. In the Earth-ionosphere cavity there are a myriad of electromagnetic natural and artificial signals produced by a huge number of sources (Palangio, 1993). The spectrum of these signals spreads twelve orders of magnitude in the frequencies domain, and fourteen orders of magnitude in the energy domain (Lanzerotti et al., 1990). Taking into account the multiplicity of the parameters that must be measured, and the complexity of the involved physical mechanisms, the level of instrumental noise needs to be so low to detect the weakest electromagnetic natural signals. The spectral density of the feeblest magnetic natural signal is about $[5-10] f T / \sqrt{\mathrm{Hz}}$ near the Earth-ionosphere cut-off frequency $(1700 \mathrm{~Hz})$. So the background noise level of the developed instrumentation must be at least $[1-2] f T / \sqrt{\mathrm{Hz}}$. Figures 1, 2 and 3 show some examples of the technology developed in the frame of this project in the field of electric and magnetic sensors. These sensors are installed in the first MEM station located in the area of L'Aquila INGV Geomagnetic Observatory $\left(42^{\circ} 23^{\prime} \mathrm{N}, 13^{\circ} 19^{\prime} \mathrm{E}, 682 \mathrm{~m}\right.$ a.s.1.). Within the middle of 2008 two new MEM observatories will be activated in Barete $\left(42^{\circ} 30^{\prime} \mathrm{N}, 13^{\circ} 16^{\prime} \mathrm{E}, 930 \mathrm{~m}\right.$ a.s.l. $)$ and Duronia $\left(41^{\circ} 39^{\prime} \mathrm{N}, 14^{\circ} 28^{\prime} \mathrm{E}, 910 \mathrm{~m}\right.$ a.s.l.). Figure 4 shows the locations in Central Italy of the first three MEM stations. In each network station will be measured the three

Published by Copernicus Publications on behalf of the European Geosciences Union. 


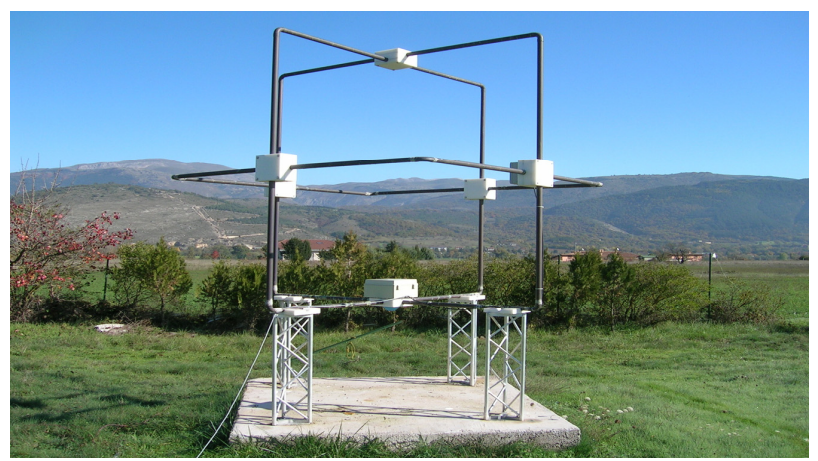

Fig. 1. Three axes induction antenna for magnetic field measurement in the frequency band $[1 \mathrm{~Hz}-100 \mathrm{kHz}]$. The instrumental sensitivity is $1 f T / \sqrt{\mathrm{Hz}}$.

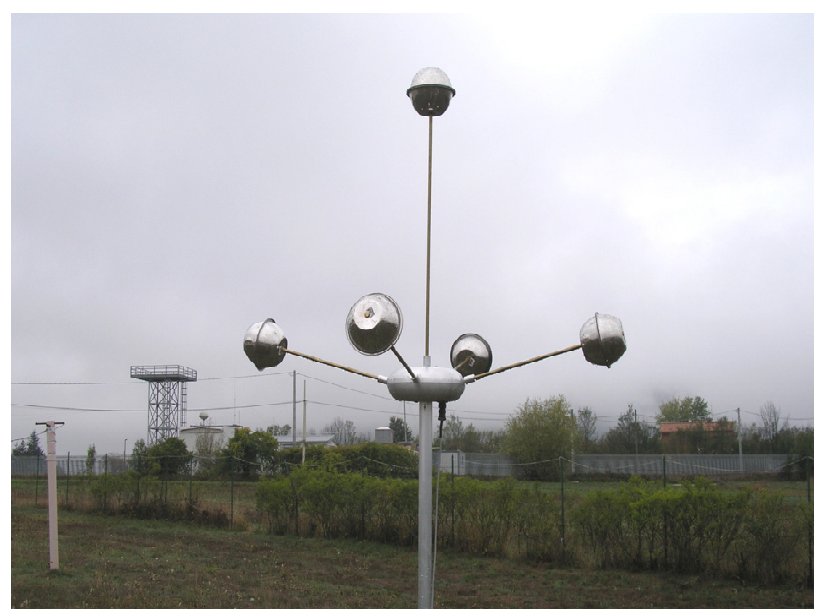

Fig. 2. Three axes antenna for electric field measurement in the frequency band $[1 \mathrm{~Hz}-100 \mathrm{kHz}]$. The instrumental sensitivity $1 \mu \mathrm{V} /(m \sqrt{\mathrm{Hz}})$.

components of the magnetic field, the three components of the electric field and the two components of the telluric field. The network configuration is needed to separate the natural electromagnetic fields from the artificial ones, and to separate the electromagnetic fields originated in the Earth's interior from those having an external source. In Figs. 5, 6, 7 and 8 are reported as examples some results obtained in first MEM station of L'Aquila. Figure 5 shows an example of the ground electric resistivity profile, calculated for L'Aquila station through the evaluation of the single station magnetotelluric tensor. The 1D profile is obtained using the conventional magnetotelluric approach. The knowledge of the underground resistivity structure is an essential requirement in order to study the electromagnetic anomalies correlated with the tectonic activity. Figure 6 shows a typical 3-D plot of the magnetic energy as function of the frequency and the time in the band ranging from $1 \mathrm{~Hz}$ to $50 \mathrm{kHz}$. In the figure can be

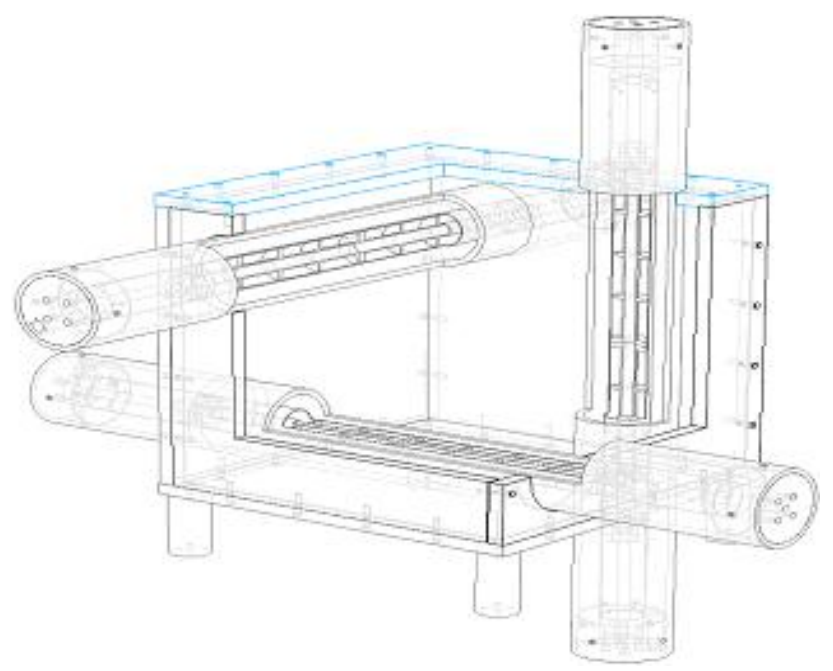

Fig. 3. Three axes search coil magnetometer for magnetic field measurements in the frequency band $[0.001-100] \mathrm{Hz}$. The instrumental sensitivity is $50 \mathrm{fT} / \sqrt{\mathrm{Hz}}$.

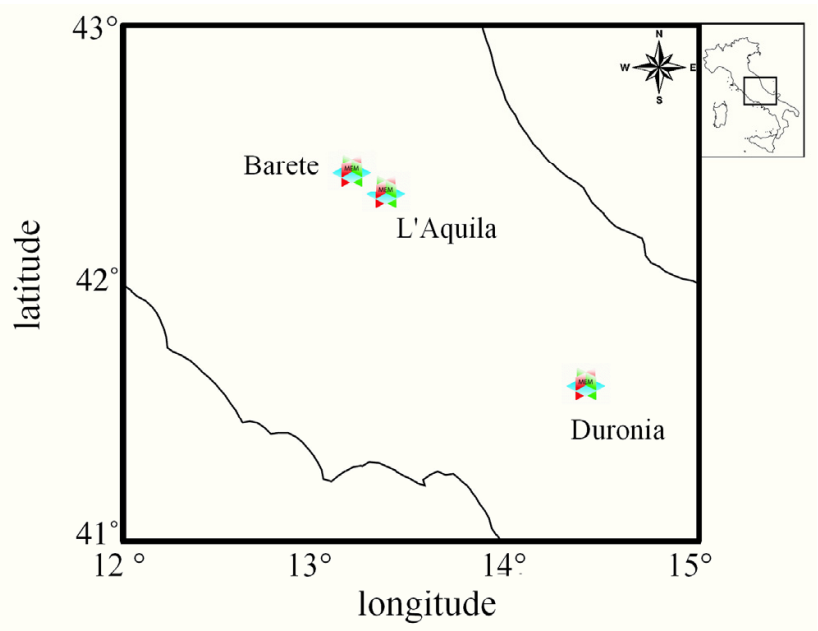

Fig. 4. The distribution in Central Italy of the first three MEM stations.

noted several artificial signals around 50-500 Hz. Moreover can be also pointed out a considerable number of quasi stationary signals modulated by the ionospheric night-day variability and a non-stationary background radiation of natural origin. Figure 7 shows an example of the 2-D representation of the horizontal magnetic field in the frequency and time domains for 12 December 2005. The plot shows stationary artificial signals $(50 \mathrm{~Hz}$ and its harmonics till few $\mathrm{kHz})$ and a lot of signals due to non stationary sources. Figure 8 shows an example of the zenithal component of the Poynting vector as function of the time and the frequency. From the figure can be noted that the arrival direction is ranging mainly from $50^{\circ}$ to $130^{\circ}$, showing that the major part of the signals reaches the measurement point by ionospheric reflection. 


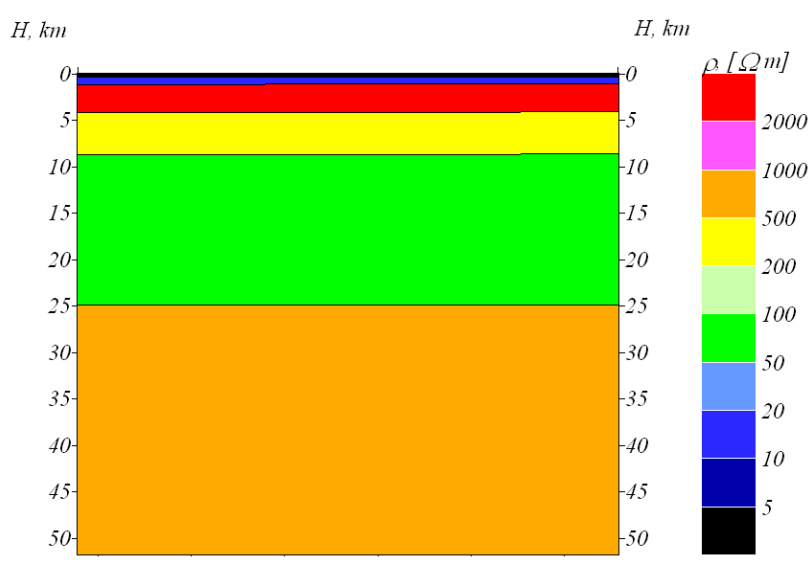

Fig. 5. 1-D resistivity model structure of the underground in the area of the Observatory of L'Aquila.

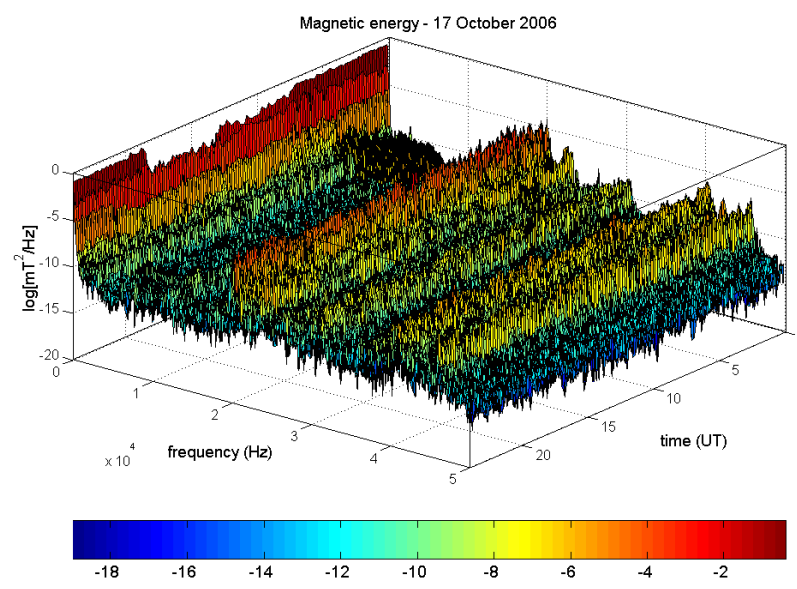

Fig. 6. Example of the magnetic field power spectral density as function of time and frequency in the band $[1 \mathrm{~Hz}-50 \mathrm{kHz}]$.

\section{The Wide band interferometry}

As previously stressed, at present only one station (L'Aquila) of the network is working. When all the stations of the network will be in operation the wide-band interferometry technique will be employed. Combining the simultaneous observations of the electromagnetic field measured in the different network stations, we will be able to obtain detailed information about the investigated electromagnetic sources. The interferometry is a technique used in different fields of the physics, as in the astronomical research. The radioastronomical interferometry is based on the simultaneous observations, in different points, of the signal emitted by a radio-source in order to study the interference pattern. The target is to obtain more information respect to the single station observation. The wide band radio-astronomical interferometry is founded on the following assumptions: 1) the source can be assumed as a point source; 2) the distances

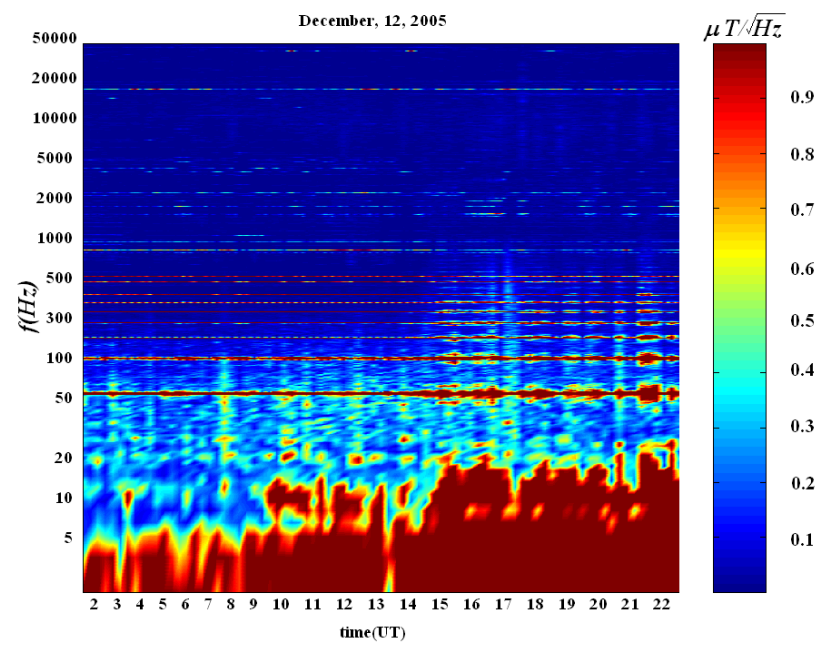

Fig. 7. Example of the horizontal magnetic field spectrogram in the frequency band $[1 \mathrm{~Hz}-50 \mathrm{kHz}]$.

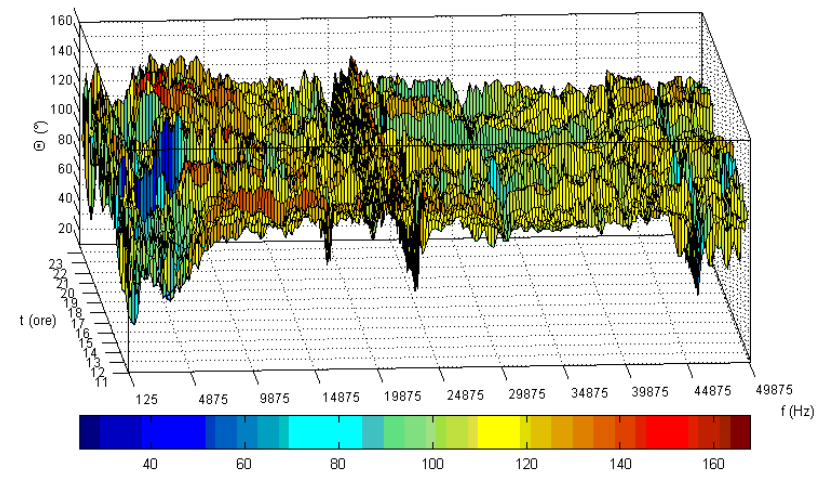

Fig. 8. Example of the zenithal component of the Poynting vector.

between the source and the measurement stations are larger than the distances between the stations; 3 ) the wavelength of the signals is smaller than the interferometric array dimension; 4) the observers are always in the far field condition; 5) the measured electromagnetic waves are observed over a finite but narrow bandwidth. In the low frequency (ULFELF-VLF) wide band interferometry, these assumptions are overturned: 1) the sources are mainly broad, their dimensions are comparable or larger with the array dimension; 2) the distances between the sources and the measurement stations are comparable with the dimension of the interferometric array; 3 ) the wavelength of the signals is larger than the interferometric array dimension; 4) the observers are always in the near field condition ; 5) the measured electromagnetic waves are observed over a wide bandwidth. The consequence of these assumptions is that an electromagnetic source is detected in each measurement stations in a different way both for its physical properties and for the statistical and spectral properties of the generated signals. This is due to the in- 
teraction of the signals with the Earth-ionosphere cavity too. The physical properties of this cavity have significant temporal and spatial variations, so that the signals simultaneously observed are different for different observation points even if the source is symmetric respect to the observation points. The observations obtained in a single point at any time, are generally different even if the source has stationary properties in a time interval larger than the measurement time step. Our principal targets are the realization of the electromagnetic tomography in the investigated area and the characterization of the signal sources detected in the interferometric array. The first target is obtained applying the Cauchy integral theorem on the interferometric array domain. The measurements are interpolated in the space to reconstruct the functions of the electromagnetic field components on the border of the interferometric array domain. The principal harmonics are reconstructed in the frequency domain by means of holomorphic functions. The second point is obtained starting from the dimensioning of time window used in the signal elaborations. This choice is based on the statistical properties of the observed signals. For each class of phenomena it is needed to choice a temporal window having local stationarity characteristic and taking into account that the background noise is mainly non stationary. The data acquisition is divided in the two bands [0.001-100] Hz and [0.001$100] \mathrm{KHz}$. In the first band the data are stored as sampled. In the second band the FFTs of the six components of the electromagnetic field are calculated in real time with $1 \mathrm{~Hz}$ resolution. Starting from the FFTs is produced an electromagnetic tensor $T(6 \times 6)$. Using the four $(3 \times 3)$ matrices of the tensor we can generate 200000 spectral matrices $(3 \times 3)$ every ten minutes. Concerning the individuation of the frequency bands related to the discrete sources, the matrices are grouped together taking into account the orthogonality criterion by the calculation of the eigenvectors and the eigenvalues. This topic is particularly interesting because, taking into account the statistical uniformity of the selected bands, we can establish the minimum number of independent sources which make a contribution to the measured noise. The energy distribution is calculated applying the Poynting vector divergence theorem, and analyzing the subclass of the selected matrices in order to evaluate, for each band, the four contributions to the energy. Since the transversal dimension of the Earth-ionosphere cavity is generally smallest than the wavelengths of the detected signals, the near field condition is dominant. The imaginary component of the Poynting vector represents, in the near field approximation, the energy of the signals propagating in the direction of the source. The surface that irradiates the signals apparently does not coincide with the physical source, but seems to coincide to a sphere with a radius $r \approx \frac{\lambda}{2 \pi}$. In the case of the natural background noise, there is an overlapping of electromagnetic fields, due to a lot of sources. The narrow band analysis allows us to evaluate approximately the different contribution to the energy, and to discriminate the signals in the relative frequency bands. These bands must be adequately narrow to reduce the overlapping of the signals originated from different sources. Moreover, the study of the wide band sources is approached by means of two matrices each of that relative to the electric field and the magnetic field of the electromagnetic tensor $T$. The two matrices can be defined in the frequency and the time domains. The analysis of the four components of the symmetric and antisymmetric parts, allows us to calculate all the parameters of the field 3D distribution. The study of the electromagnetic tensor derivative allows us to separate the variation of the electromagnetic field in four components. The first two are parallel and orthogonal to the electric field; the other two are parallel and orthogonal to the magnetic field. The environmental electromagnetic field analysis must take into account that the signals propagate in a resonant cavity which is characterized by free resonance modes, both longitudinal and transversal, and cut-off frequencies relative to the TM and TE modes (Lazebnyy et al., 1988). The complexity of the electromagnetic field observable on the Earth surface in the band $[0.001 \mathrm{~Hz}-100 \mathrm{kHz}]$ suggests to apply some simplifications by means of a schematization of the sources of the cavity. These sources can be approached with an elementary dipole more or less widespread, or with electrical and magnetic multipoles. The emitted radiation can assume thermic, coloured or monochromatic characteristics. From statistics point of view the sources can be gaussian, isotropic, stationary, impulsive and so on. In nature we can observe different combinations of these schematizations. There are also electromagnetic sources not radiating or rather (QNR); this kind of sources is common enough. Their electromagnetic fields remain relegated into the source volume. A typical example are the toroidal source as the dynamo sources present in the magnetosphere, ionosphere and in the Earth core. Generally the large complex sources have a toroidal component. This component cannot be measured faraway from the source; it is impossible to define the energy balance of the system. Only the poloidal components can be measured. In the case of extended 3-D sources having dimensions larger than the principal wavelength, the poloidal components produce electric and magnetic fields which temporal and spatial correlation lengths reflects the coherence properties of the source directly connected with the mechanism of fields generation.

\section{Conclusions}

The scientific and the technological objectives of the MEM project are reported particularly regarding the monitoring of the electromagnetic signals originated in the Earth's interior that can be associated with the seismic activity. Some examples of the technology developed in the frame of the project are also reported. We have also shown the preliminary results obtained in the first MEM station installed in the area of the INGV Observatory of L'Aquila. The wide 
band interferometry is proposed as innovative technique to obtain detailed information about the different electromagnetic sources which contribute to the environmental electromagnetic signals. Combining the simultaneous observation of the electromagnetic field measured in the stations of the network we will be able to obtain information about the different electromagnetic sources. At present the network of observatories does not exist, only L'Aquila Observatory is working. Within the middle of 2008 two new stations will be added to the network. When all the observatories will be in operation the wide band interferometry technique will be applied.

Acknowledgements. The authors thank the staff of the INGV L'Aquila Observatory for the basic support in the research activity. This work was supported by the MEM Project (Interreg IIIA Adriatic Cross Border Programme).

Edited by: P. Fabian

Reviewed by: two anonymous referees

\section{References}

Fraser-Smith, A. C., Bernardi, A., McGill, P. R., Ladd, M. E., Helliwell, R. A., and Villard Jr., O. G.: Low Frequency Magnetic Field Measurements near the Epicenter of the Ms 7.1 Loma Prieta Earthquake, Geophys. Res. Lett., 17, 1465-1468, 1990.

Lanzerotti, L. J., Maclennan, C. G., and Fraser-Smith, A. C.: Background magnetic spectra: $\sim 10 \mathrm{E}-5$ to $\sim 10 \mathrm{E}+5 \mathrm{~Hz}$, Geophys. Res Lett., 17, 10, 1593-1596, 1990.

Lazebnyy, B. V., Nikolayenko, A. P., Rafal'skiy, V. A., and Shvets A. V.: Detection of Transverse Resonances of the EarthIonosphere Cavity in the Average Spectrum of VLF Atmospherics, Geomagn. Aeronomy+, 28, 2, 281-282, 1988.

Palangio, P.: Radioricezione ELF-VLF, Ann. Geofis., 36, 5-6, 99114, 1993.

Palangio, P., Di Lorenzo, C., Masci, F., and Di Persio, M.: The study of the electromagnetic anomalies linked with the Earth's crustal activity in the frequency band $[0.001 \mathrm{~Hz}-100 \mathrm{kHz}]$, Nat. Hazards Earth Syst. Sci., 7, 507-511, 2007, http://www.nat-hazards-earth-syst-sci.net/7/507/2007/.

Teisseyre, R. and Ernst, T.: Electromagnetic radiation related to dislocation dynamics in a seismic preparation zone, Ann. Geophis. Italy, 45, 393-399, 2002. 